\title{
A Regression Neural Network based Glaucoma Detection System using Texture Features
}

\author{
Swapna P.P. ${ }^{1}$, and Mini M.G ${ }^{2}$
}

\begin{abstract}
Glaucoma detection and classification has been an area of prime significance because of its numerous applications in the field of healthcare and medical engineering. The suitable selection of image specific features is one of the major factors determining the classification efficiency of any system. Glaucoma detection using eye fundus images necessitates careful extraction of texture features to be utilized in the training of the classifier architecture. In this paper, a novel method is proposed, making use of a combined feature set of Fractal Dimension calculated from extracted texture feature of total fundus images and Local Binary Pattern (LBP) feature along with an efficient Regression Neural Network decision making unit. An accuracy of $88.70 \%$ has been obtained by the proposed system, with sensitivity of $87.20 \%$ and a specificity of $90 \%$.
\end{abstract}

Keywords - Eye fundus, Fractal Dimension, Local Binary Pattern, Regression Neural Network.

\section{INTRODUCTION}

$\mathrm{G}^{\mathrm{s}}$ LAUCOMA detection and classification have got immense relevance in the field of medical engineering. Glaucoma is an eye disorder occurring due to irreversible progressive damage of optic nerve that leads to loss of vision. It is the second cause for blindness in world after refractive and cataract errors [1]. The optic disc is a probable area for effective diagnosis of glaucoma. Damage due to glaucoma is irreversible, but its progression can be retarded if early diagnosis and treatment are carried out. Fundus images of eyes can be utilized effectively in the retinal analysis, as reported in open literature.Methods have been proposed glaucoma detection making use of generic feature types, thereby extracting a Risk Index Parameter[2], extracted energy signatures by 2D wavelet transform analysis [3], and Hough Transform and Pyramidal Decomposition [4].

In this paper, an image processing based approach for automated glaucoma detection has been proposed, utilizing a modified texture feature extraction and an efficient Regression Neural Network architecture. The eye fundus images of sufficient resolution have been utilized in the analysis, for the extraction of texture features. The proposed system makes use of extracted Fractal Dimension of the eye fundus. In this approach, in addition to optic disc, which is

\footnotetext{
${ }^{1}$ Department of Electronics Engineering,Model Engineering College,Kochi21, India

${ }^{2}$ College of Engineering, Cherthala, Alappuzha,India.
}

the primary location for glaucoma detection, the probability of structural changes in other areas of eye fundus has also been considered. For better detection performance and accuracy, Local binary pattern (LBP) based feature extraction has also been carried out, specifically for the optical disc area in eye fundus images. This Region of Interest (ROI) based approach for LBP feature extraction, in addition to the totalistic approach adopted for Fractal Dimension features of the eye fundus, can effect significant improvement in glaucoma detection with moderate implementation complexity.

\section{THEORETICAL ASPECT}

In this work, two algorithms are proposed for feature extraction based on Fractal Dimension and Local Binary Pattern. Figure 1 demonstrates a typical fundus image infected with glaucoma, along with a normal eye fundus image.



Fig. 1. a) Glaucomated fundus image b) Healthy fundus image

\subsection{Fractal Dimension}

The texture property of digital images can be analyzed using Fractal Dimension. It provides a numerical measure of the image by box counting algorithm for detecting the complexity of the spatial and temporal patterns. The algorithm analyses the patterns by decomposing the image into box shaped pieces and the process is repeated with smaller scales for accurately describing the structure of patterns [5].

In box counting method, the image is overlaid with a grid and then the number of boxes covering the entire image with at least one pixel element is counted. The size of the grid is successively reduced to finer boxes. In this algorithm, fractal dimension is calculated by taking the ratio.

$$
D=\frac{\log (N)}{\log \left(\frac{1}{r}\right)}
$$


Where $N$ is the number of boxes and $1 / r$ is the magnification or inverse of the box size.A graph can be plotted by taking $\log N$ on y-axis and $\log 1 / r$ on $\mathrm{x}$-axis. The slope of the graph gives the fractal dimension. This extracted Fractal Dimension can be utilized in the training of the Regression Neural Network architecture.

\subsection{Local Binary Pattern Based Features}

The neurodegeneration of the optic nerve is predominant in and around the optic disc region. Similarly retinal nerve fibre layer degeneration in the peripappillary area can be observed as bright region around the optic disc [6]. The optic disc and peripappillary area are appeared as the brightest regions in the color retinal images. The PPA refers to peripappillary atrophy which is a pathological indicator associated with myopia and Glaucoma[7]. PPA is the degeneration of the retinal pigment epithelial layer and photoreceptors in the region surrounding the optic nerve head. Altered position of blood vessels in the optic disc, disc hemorrhages and presence of PPA are important indicators and effectiveness of treatment depends on accurate detection at its early stages.

Local Binary Pattern (LBP) is a powerful descriptor for texture which converts an image into an array of integers or histogram bins to describe local textural patterns [8]. The basic LBP is obtained by summing the threshold difference, weighted by powers of 2 . The $\mathrm{LBP}_{\mathrm{PR}}$ code of a pixel $\left(\mathrm{x}_{\mathrm{c}}, \mathrm{y}_{\mathrm{c}}\right)$ is defined as

$$
L B P_{P R}\left(x_{c}, y_{c}\right)=\sum_{P=0}^{P-1} s\left(g_{p}-g_{c}\right) 2^{P}
$$

Where $g_{c}$ and $g_{p}$ are the gray values of the centre pixel and its neighbors, $P$ is the number of neighbors, $R$ is the radius of the local neighborhood and $s(z)$ is the thresholding function

$$
\begin{array}{cc}
s(z)=1 & z \geq 0 \\
=0 & z<0
\end{array}
$$

Neighborhood is a set of pixels $P$, lying on a circle of radius $R$. The derived LBP values statistically describe the image characteristics.

\subsection{Regression Neural Network Architecture}

General Regression Neural Network (GRNN) is used in applications for estimation of continuous variables through quick learning [9]. They are multilayer feed forward neural network with highly parallel structure. The architecture of a Regression Neural Network is shown in figure 2. The input sample vector $\mathrm{X}$ be applied to input units. The input units are distribution units. The probability density function used in GRNN is the normal distribution. Each training sample $X_{i}$ is used as the mean of the normal distribution. $A^{i}$ and $B^{i}$ are adaptive weights in the summation layer. The network consists of four layers-input layer, pattern layer, summation layer and output layer

$$
\hat{Y}(X)=\frac{\sum_{i=1}^{n} Y^{i} \exp \left(\frac{-D i^{2}}{2 \sigma^{2}}\right)}{\sum_{i=1}^{n} \exp \left(\frac{-D i^{2}}{2 \sigma^{2}}\right)}
$$

Where scalar function

$$
D i^{2}=\left(X-X^{i}\right)^{T}\left(X-X^{i}\right)
$$

The estimated $\hat{Y}(X)$ is a weighted average of all observed values $Y^{i}, \sigma$ is the smoothing parameter or spread value.

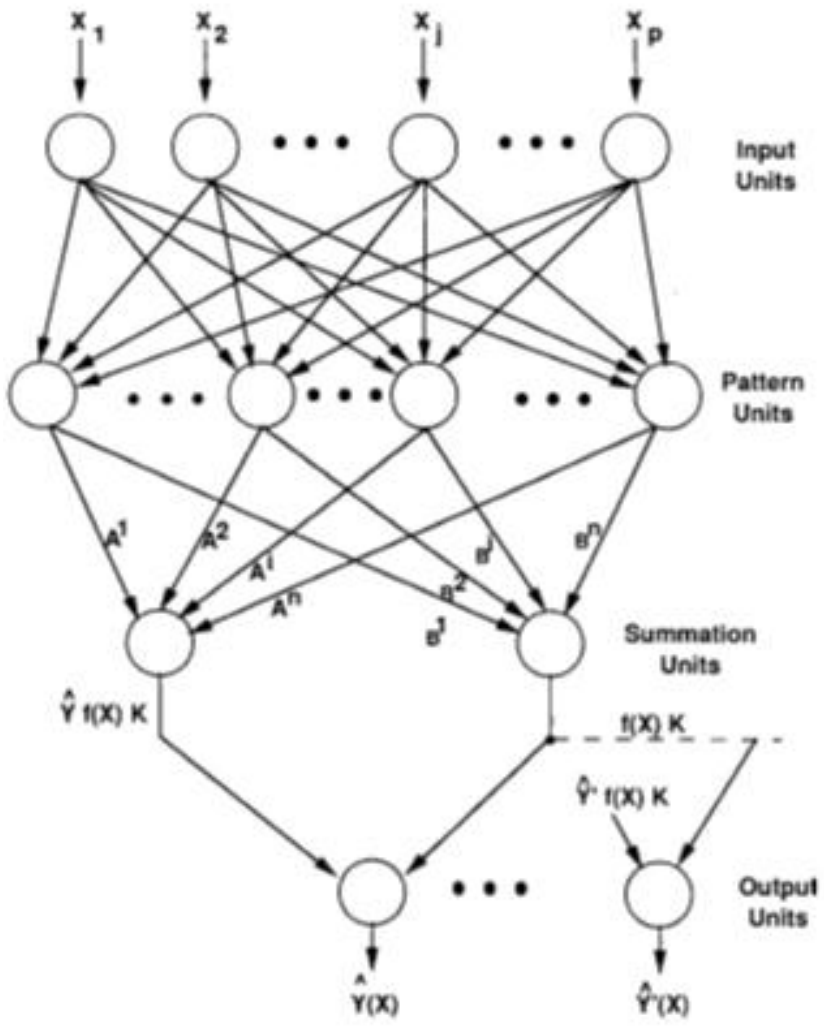

Fig. 2. General regression neural network architecture [9]

\section{Proposed Glaucoma Detection System}

The proposed system consists of a preprocessing stage followed by ROI selection, feature extraction and finally a neural network based classifier. Figure 3 depicts the flow diagram of the proposed glaucoma detection system. Preprocessing has been carried out for extraction of fractal dimension feature from the eye fundus image. ROI based LBP feature extraction is also done, resulting in a more efficient computation. Efficient neural network architecture is made use of in the proposed Glaucoma detection system. For the color retinal fundus images made used in the system, the ROI is obtained by approximating centre as optic disc and from this region the LBP feature values are taken. In addition to that, another set of Fractal Dimension texture feature is extracted from the totalistic fundus images. These feature sets are utilized for training the Regression based Neural Network architecture for classification. When suitably formulated and trained, the decision making architecture carries out detection 
and classification of Glaucoma affected eye fundus images.

\subsection{Data Collection}

In this approach, 80 eye fundus images have been considered for Glaucoma detection. The images are collected from open domain and research groups. Fifty images were provided by a research group Drishti GS in connection with Aravind eye hospital with resolution 2896 x 1944 pixels in PNG format [10][11]. Another 30 images taken for training were downloaded from open domain [12]. The extracted features were made use for classification.

For quantitative analysis of the system performance, the following metrics [13] can be made use of. The terms commonly used along with sensitivity, specificity and accuracy are described in terms of TP (True Positive), TN (True Negative), FN (False Negative) and FP (False Positive).

Sensitivity $=\mathrm{TP} /(\mathrm{TP}+\mathrm{FN})=($ Number of true positive assessment) / ( Number of all positive assessment)

Specificity $=\mathrm{TN} /(\mathrm{TN}+\mathrm{FP})=($ Number of true negative assessment) / (Number of all negative assessment)

Accuracy $=(\mathrm{TN}+\mathrm{TP}) /(\mathrm{TN}+\mathrm{TP}+\mathrm{FN}+\mathrm{FP})=($ Number of correct assessments) / Number of all assessments)

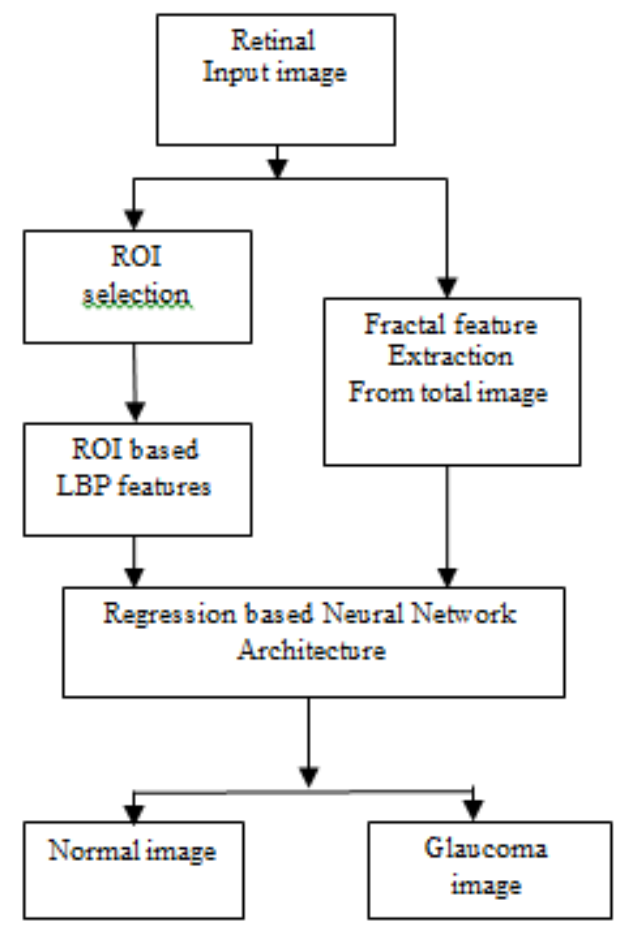

Fig. 3. Flow diagram of the proposed system

\section{Simulation Results}

The system performance has been validated using simulation studies. The proposed glaucoma detection system has been tested for 80 eye fundus images. The pre-processed fundus images are utilized in the extraction of texture features as proposed. Figure 4 shows a typical extracted Fractal Dimension which can be obtained from the slope of the graph. The pathological changes in the optic disc area, presence of haemorrhages and PPA are important parameters in Glaucoma detection. By defining the ROI to only focus on optic disc and its periphery, errors resulting from other image artifacts can be minimized.

Figure 5 shows distribution of LBP feature values of a typical Glaucoma affected fundus image and healthy image respectively. The feature vector consists of nine extracted elements, representing three different neighbourhoods with radius 1,2 and 4 .

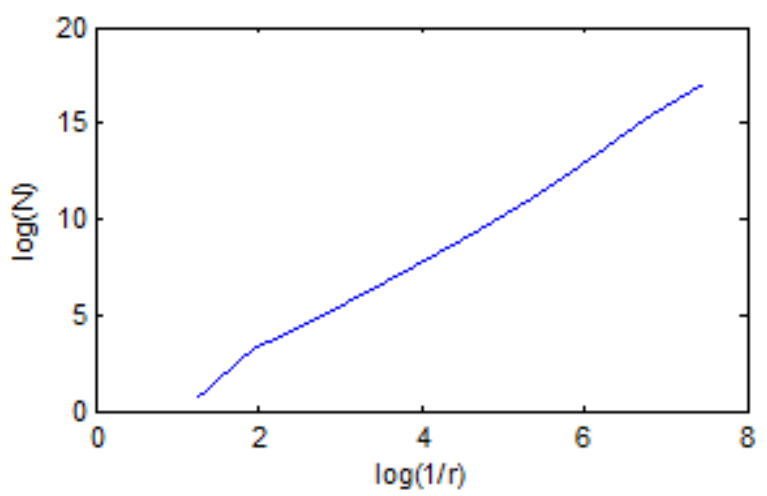

Fig. 4. Plot of $\log \mathrm{N}$ vs $\log 1 / \mathrm{r}$ of a fundus image

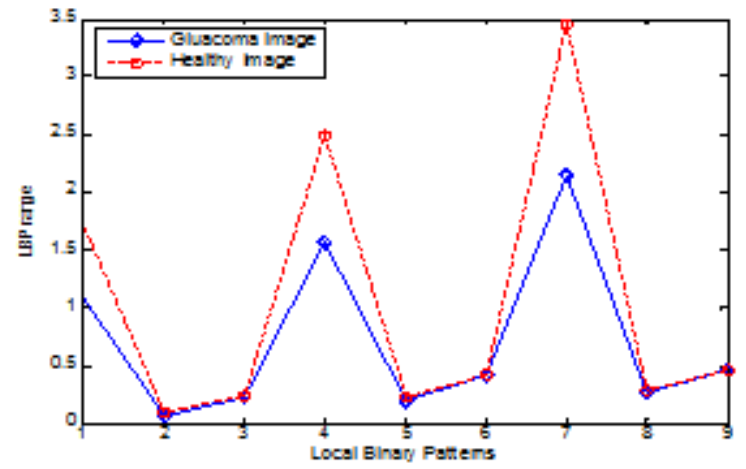

Fig.5. Typical extracted LBP values for Glaucomatous and Healthy images

The GRNN classifies these extracted features to identify the normal and Glaucoma fundus images according to the texture based features. Experiments have been carried out to verify the ability of the GRNN in achieving good classification rate. The system classification capability has been quantitatively analyzed using the set of eye fundus images and a detection rate of the $88.70 \%$ has been obtained. The classification capability can be further increased by the augmentation with more feature types, extended training, and by improvements in decision making architecture. The table below summarizes the detection rate obtained by the proposed Glaucoma detection system.

TABLE I

Quantitative Analysis Of The Proposed Glaucoma Detection System

\begin{tabular}{|c|c|c|c|}
\hline $\begin{array}{c}\text { No of images } \\
\text { tested }\end{array}$ & Sensitivity & Specificity & Accuracy \\
\hline 80 & $87.2 \%$ & $90 \%$ & $88.7 \%$ \\
\hline
\end{tabular}

\section{V.CONCLUSION}

An effective glaucoma classifying system has been proposed, making use of a modified feature extraction 
technique. The approach made use of totalistic Fractal Dimension and ROI based LBP based feature extractions. In addition, an efficient Regression Neural Network based classifying architecture is also made use of by the proposed system. Simulation results demonstrated the effectiveness of the system, in the field of glaucoma detection and classification.

\section{ACKNOWLEDGMENT}

The authors gratefully acknowledge the support extended to this research work by Model Engineering College, Ernakulam and College of Engineering, Cherthala, India.

\section{REFERENCES}

[1] Malay, K. D., Amit, K. M., Anushikha, S., Parthasarathi, M, Radim, B, and Kamil, R." Glaucoma Detection by Segmenting the Super Pixels from Fundus Colour Retinal Images". In Proceedings of the International Co,nference on Medical Imaging, m-Health and Emerging Communication Systems (MedCom) 978-1-4799-5097-3/14 IEEE 2014.

[2] Rüdiger, B., Jörg, M., László, G. N., Joachim, H, and Georg, M.’ Glaucoma risk index: Automated glaucoma detection from color fundus images", Medical Image Analysis Elsevier B.V. 14 pp, 471-481. DOI http://dx.doi.org:10.1016/j.media.2009.12.006. 2010

[3] Sumeet Dua, Rajendra Acharya, U. Pradeep, C. and Vinitha Sree, S "Wavelet-Based Energy Features for Glaucomatous Image Classification", IEEE Trans. On Information Technology in Biomedicine. Vol. 16, No. 1, January 2012. 1089-7771 DOI= http://dx.doi.org/10.1109/TITB.2011.2176540.

[4] Caroline Viola, M. and Jainudhin Sudar, B. " Automatic Optic Nerve Head Segmentation for Glaucomatous Detection using Hough Transform and Pyramidal Decomposition", In Proceedings of the International Conference on Recent Trends in Computational Methods, Communication and Controls (ICON3C 2012) International Journal of Computer Applications (IJCA).

[5] Paul, Y. K. Khan, M. I. Pinakin, G. D. M'arta, T. Anita, G. Gabor, H. and Edward, A. E," Novel fractal feature-based multiclass glaucoma detection and progression prediction", IEEE J. of Biomedical and Health Informatics, Vol. 17, No. 2, March 2013. 2168-2194 DOI= http://10.1109/TITB.2012.2218661.

[6] Cheng, K. L. Tong Boon, T. Augustinus, L. Baljean, D. and Alan, Murray, F," Parapapillary atrophy and optic disc region assessment (PANDORA): retinal imaging tool for assessment of the optic disc and parapapillary atrophy", .J. of Biomedical Optics 17(10). (October 2012). DOI= http://dx.doi.org/10.1117/1.JBO.17.10.106010.

[7] Jiang, L. Damon, W. K. W. Joo Hwee, L. Ngan Meng, T .Zhuo Zhang, Huiqi, L. Fengshou, Y. Benghai, L. Seang, M. S. Louis, T. and Tien, Y. W," Detection of Pathological Myopia by PAMELA with Texture-Based Features through an SVM Approach", . J. of Healthcare Engineering • Vol. 1 - No. 1 pp 1-11 • 2010. DOI= http://dx.doi.org/10.1260/20402295.1.1.1.

[8] Ali Mohsin, Al-juboori, Wei Bu, Xiangqian, W. and Qiushi, Z. "Palm Vein Verification Using Multiple Features and Isometric Projection", International Journal of Signal Processing, Image Processing and Pattern $\begin{array}{llll}\text { Recognition Vol.7 No.1 (2014). pp.33-44. DOI= } & \end{array}$ http://dx.doi.org/10.14257/ijsip.2014.7.1.04.

[9] Donald, F. Specht 1991. “A general regression neural network”, IEEE Tranc. On Neural networks. Vol 2. No.6. November 1991.

[10] http://cvit.iiit.ac.in/projects/mip/drishti-gs/mipdataset2/Home.php Provided by Medical Image Processing (MIP) group, IIIT Hyderabad.

[11] Sivaswamy, J., Krishnadas, S. R, Chakravarty A, Joshi, G.D, Ujjwal. "A Comprehensive Retinal Image Dataset for the Assessment of Glaucoma from the Optic Nerve Head Analysis", JSM Biomed Imaging Data Pap 2(1): 1004. 2015 .

[12] http://www5.cs.fau.de/research/data/fundus-images.

[13] Annu, N., Justin, J. "Automated Classification of Glaucoma Images by Wavelet Energy Features", International Journal of Engineering and Technology (IJET). International Journal of Engineering and Technology (IJET) Vol5 No2 pp 1716-1721. 2013. 